Pacific Journal of Mathematics

REPRESENTATION OF SUPERHARMONIC FUNCTIONS MEAN
CONTINUOUS AT THE BOUNDARY OF THE UNIT BALL 


\title{
REPRESENTATION OF SUPERHARMONIC FUNCTIONS MEAN CONTINUOUS AT THE BOUNDARY OF THE UNIT BALL
}

\author{
J. R. DIEDERICH
}

\begin{abstract}
In this paper it will be shown that superharmonic functions can be represented by a Green potential together with their boundary values if taken mean continuously at the boundary of the unit ball.
\end{abstract}

Introduction. It is well known that if $u(r, \theta, \phi)$ is harmonic inside the unit ball and has radial $\operatorname{limit}_{\lim } u(r, \theta, \phi)=0$ everywhere on the surface, then $u$ is not necessarily identically null inside and thus cannot be represented by its radial boundary values. Furthermore, there is an $L_{1}$ (Lebesgue class) harmonic function, see $\S 2$. Remarks, which satisfies $\lim _{r \rightarrow 1} u(r, \theta, \phi)=0$ except for $(1,0,0)$. In [1] and [3], Shapiro established the representation of harmonic functions in the two dimensional unit disc by their radial limits when a certain radial growth condition is satisfied. However, the set of functions satisfying the radial growth condition does not contain the class $L_{1}$, and conversely. Also, the analogues of [1] and [3] have not been established in the $N$-dimensional unit ball, $3 \leqq N$.

Our intention is to establish a representation of superharmonic functions in $L_{1}$ on the $N$-dimensional unit ball by their boundary values if taken mean continuously. Definitions and the statement of the theorems follow in the next section.

1. Preliminaries. We shall work in $N$-dimensional Euclidean space $R^{N}, 3 \leqq N$, and shall use the following notation: $x=\left(x_{1}, \cdots\right.$, $\left.x_{N}\right)$ and $B(x, r)=$ the open $N$-ball centered at $x$ with radius $r ; \widetilde{B}(x$, $r)=B(x, r) \cap B(0,1) ;|E|$, the Lebesgue measure of $E ; \partial E$, the boundary of $E ; \bar{\partial} B(x, r)=\partial B(0,1) \cap B(x, r) ; d \omega_{N}$, the natural surface area on $\partial B(0,1)$; and subscripted $A$ 's, positive absolute constances though possibly different from one occurrence to another. For a point $y_{0} \in$ $\partial B(0,1), u(x)$ a measurable function on some $\widetilde{B}\left(y_{0}, r_{0}\right)$, and $f(y)$ a function on $\partial B(0,1)$, we set for $\rho \leqq r_{0}$

$$
u_{f}\left(y_{0}, \rho\right)=\left|\widetilde{B}\left(y_{0}, \rho\right)\right|^{-1} \int_{\widetilde{B}\left(y_{0}, \rho\right)}\left|u(x)-f\left(y_{0}\right)\right| d x .
$$

We use the notation $u\left(y_{0}, \rho\right)$ when $f \equiv 0$.

THEOREM 1. Let $u(x)$ be superharmonic in $\Omega=B(0,1)$. If 


$$
\begin{gathered}
u(y, \rho)=O(1) \text { as } \rho \longrightarrow 0 \text { for each } y \in \partial \Omega \\
u(y, \rho)=o(1) \text { as } \rho \longrightarrow 0 \text { a.e. }[d \omega] \text { on } \partial \Omega
\end{gathered}
$$

then $0 \leqq u(x)$ on $\Omega$.

Theorem 1 is the main step in establishing

THEOREM 2. Let $u(x)$ be superharmonic in $B(0,1)$. Let $f(y)$ be in $L_{1}$ on $\partial \Omega$ and satisfy

$$
\begin{aligned}
\int_{\bar{\partial}_{B\left(y_{0}, \rho\right)}}\left|f(y)-f\left(y_{0}\right)\right| d \omega_{N}(y) & =O\left(\rho^{N-1}\right) \\
\text { as } \rho \longrightarrow 0 \text { for each } & y_{0} \in \partial \Omega .
\end{aligned}
$$

If $u_{f}(y, \rho)$ satisfies (1) and (2), then

$$
u(x)=\int_{\Omega} G\left(x, x^{\prime}\right) d \eta\left(x^{\prime}\right)+P I(f, x)
$$

where $G\left(x, x^{\prime}\right)$ is the Green function for $\Omega, \eta$ is a nonnegative additive measure on $\Omega$, and $P I(f, x)$ is the Poisson integral of $f$.

2. REMARK. Theorem 1 is best possible in two respects. If (1) is required for all but one $y_{0} \in \partial B(0,1)$, then the conclusion fails as is demonstrated by $u(x)=\left(|x|^{2}-1\right)\left[\omega_{N}\left|x-y_{0}\right|^{N}\right]^{-1}$, with $y_{0}=(1,0, \cdots$, $0)$. Secondly, if the modulus is eliminated in the definition of $u(y, \rho)$ and the integral is defined improperly, then the conclusion fails even if (2) is strengthened to "for each $y \in \partial Q$ ". Simply consider a nonradial partial of the above function. In Theorem 2 the necessity of (3) is not clear.

Clearly, Theorem 1 offers a uniqueness theorem for harmonic functions which are mean continuous at the boundary of the unit ball. Also, contained in the proof of Theorem 1 is a generalization of the reflection principle for harmonic functions.

Finally, an open question regarding a converse to Theorem 1 will be considered in $\S 5$.

3. Proof of Theorem 1. Set $u^{-}(x)=\min (u(x), 0)$. Then $u^{-}(x)$ is superharmonic and clearly satisfies both (1) and (2). We intend, of course, to show that $u^{-}(x) \equiv 0$ which we shall do in the following steps.

Let $Z$ be the set of points $z$ on $\partial \Omega$ such that $u^{-}(x)$ is unbounded in every neighborhood $\widetilde{B}(z, \rho) . \quad \partial \Omega-Z$ clearly open so that $Z$ is a closed set.

Step 1. If $y_{0} \in \partial \Omega$ and $\bar{\partial} B\left(y_{0}, 2 \rho_{0}\right) \cap Z=\phi$, then $\lim _{x \rightarrow y} u^{-}(x)=0$ for 
$x \in \widetilde{B}\left(y_{0}, \rho_{0}\right)$ and $y \in \bar{\partial} B\left(y_{0}, \rho_{0}\right)$.

Proof. Let $y$ be a point of $\bar{\partial} B\left(y_{0}, \rho_{0}\right)$ for which (2) is satisfied. Let $x$ be a point on the line segment $l_{y}$ through the center of $\Omega$ and $y$. Select $\rho_{x}=|x-y|$, then by the superharmonicity of $u^{-}(x)$

$$
\begin{aligned}
0 & \geqq u^{-}(x) \geqq\left|B\left(x, \rho_{x}\right)\right|^{-1} \int_{B\left(x, \rho_{x}\right)} u^{-}\left(x^{\prime}\right) d x^{\prime} \\
& \geqq\left|B\left(x, \rho_{x}\right)\right|^{-1} \int_{\widetilde{B}\left(y, 2 \rho_{x}\right)} u^{-}\left(x^{\prime}\right) d x^{\prime} \\
& \geqq 2^{N}\left|\widetilde{B}\left(y, 2 \rho_{x}\right)\right|^{-1} \int_{\widetilde{B}\left(y, 2 \rho_{x}\right)} u^{-}\left(x^{\prime}\right) d x^{\prime} \\
& =-2^{N} u^{-}\left(y, 2 \rho_{x}\right) .
\end{aligned}
$$

As $x \rightarrow y, 2 \rho_{x} \rightarrow 0$, thus $u^{-}\left(y, 2 \rho_{x}\right) \rightarrow 0$, since $y$ is selected to satisfy (2). So

$$
\lim _{\substack{x \rightarrow y \\ x \in l_{y}}} u^{-}(x)=0 \quad \text { a.e. on } \bar{\partial} B\left(y_{0}, 2 \rho_{0}\right) \text {. }
$$

By the definition of $Z$ and the superharmonicity of $u^{-}(x)$ it is clear that $v^{-}(x)$ is bounded in $\widetilde{B}\left(y_{0}, \rho_{0}\right)$, and hence can be represented

$$
u^{-}(x)=\int_{\tilde{B}\left(y_{0}, \rho_{0}\right)} G_{0}\left(x, x^{\prime}\right) d \eta_{0}\left(x^{\prime}\right)+h^{-}(x)
$$

where $G_{0}\left(x, x^{\prime}\right)$ is the Green function for $\widetilde{B}\left(y_{0}, \rho_{0}\right), \eta_{0}$ is a nonnegative set function and $h^{-}(x)$ is the greatest harmonic minorant of $u^{-}(x)$. By Theorem 1 [4, p. 527], we have that

$$
\lim _{\substack{x \rightarrow y \\ x \in l_{y}}} \int_{\tilde{B}\left(y_{0}, \rho_{0}\right)} G_{0}\left(x, x^{\prime}\right) d \eta_{0}\left(x^{\prime}\right)=0 \text { a.e. on } \bar{\partial} B\left(y_{0}, \rho_{0}\right) \text {. }
$$

By this and (5)

$$
\lim _{\substack{x \rightarrow y \\ x \in l_{y}}} h^{-}(x)=0 \text { a.e. on } \bar{\partial} B\left(y_{0}, \rho_{0}\right) \text {. }
$$

Clearly $h^{-}(x)$ is bounded in $\widetilde{B}\left(y_{0}, \rho_{0}\right)$ and therefore can be represented by its radial limits. Hence $\lim _{x \rightarrow y} h^{-}(x)=0$ for $x \in \widetilde{B}\left(y_{0}, \rho_{0}\right)$ and $y \in$ $\bar{\partial} B\left(y_{0}, \rho_{0}\right)$. Since $0 \geqq u^{-}(x) \geqq h^{-}(x)$, the desired conclusion follows.

As an immediate consequence of Step 1, we have

Step 2. If $\bar{\partial} B\left(y_{0}, 2 \rho_{0}\right) \cap Z=\phi$, then the function $u_{0}^{-}(x)=u^{-}(x)$ for $x \in \widetilde{B}\left(y_{0}, \rho_{0}\right), u_{0}^{-}(x) \equiv 0$ for $x \in B\left(y_{0}, \rho_{0}\right)-\widetilde{B}\left(y_{0}, \rho_{0}\right)$ is superharmonic in $B\left(y_{0}, \rho_{0}\right)$.

Proof. $u^{-}(x)$ is continuously 0 at $\bar{\partial} B\left(y_{0}, \rho_{0}\right)$ and nonpositive in 
$\widetilde{B}\left(y_{0}, \rho_{0}\right)$.

Step 3. If $Z \neq \phi$, then there is a $z_{0} \in Z$, an $r_{0}>0$, and a constant $A_{1}$, such that

$$
u^{-}(z, \rho) \leqq A_{1} \quad \text { for } \quad z \in \bar{\partial} B\left(z_{0}, 2 r_{0}\right) \cap Z \quad(0<\rho<1) .
$$

Proof. Since $u^{-}(x)$ is superharmonic and satisfies (2), it is in $L_{1}$ on $\Omega$. Consequently by continuity of the integral $u(y, \rho)$ is jointly continuous for $0<\rho<1$ and $y \in \partial \Omega$. Proceeding as in [2, p. 69] and again employing (2) the conclusion (7) follows.

By Step 1, the conclusion of Theorem 1 follows immediately if $Z=\phi$. Assuming $Z \neq \phi$, select $z_{0}$ as in Step 3. Let $x_{1}$ be an arbitrary point in $\widetilde{B}\left(z_{0}, r_{0}\right)$, and let $\rho_{x_{1}}$ be the largest value for which $B\left(x_{1}\right.$, $\left.2 \rho_{x_{1}}\right) \cap Z=\phi$. Clearly there is a point $z^{*}$ which lies in $\bar{\partial} B\left(z_{0}, 2 r_{0}\right)$ and is on the boundary of $B\left(x_{1}, 2 \rho_{x_{1}}\right)$. By Step 2, we can extend $u^{-}(x)$ by $u_{0}^{-}(x)$ in the part of $B\left(x_{1}, \rho_{x_{1}}\right)$ lying outside $\Omega$. So

$$
\begin{aligned}
u^{-}\left(x_{1}\right) & =u_{0}^{-}\left(x_{1}\right) \geqq\left|B\left(x_{1}, \rho_{x_{1}}\right)\right|^{-1} \int_{B\left(x_{1}, \rho_{x_{1}}\right)} u_{0}^{-}\left(x^{\prime}\right) d x^{\prime} \\
& =\left|B\left(x_{1}, \rho_{x_{1}}\right)\right|^{-1} \int_{\widetilde{B}\left(x_{1}, \rho_{x_{1}}\right)} u^{-}\left(x^{\prime}\right) d x^{\prime} \\
& \geqq A_{0}\left|\widetilde{B}\left(x_{1}, \rho_{x_{1}}\right)\right|^{-1} \int_{\widetilde{B}\left(x_{1}, \rho_{x_{1}}\right)} u^{-}\left(x^{\prime}\right) d x^{\prime} \\
& \geqq 4^{N} A_{0}\left|\widetilde{B}\left(z^{*}, 4 \rho_{x_{1}}\right)\right|^{-1} \int_{\widetilde{B}\left(z^{*}, 4 \rho_{x_{1}}\right)} u^{-}\left(x^{\prime}\right) d x^{\prime} \\
& =-4^{N} A_{0} u^{-}\left(z^{*}, 4 \rho_{x_{1}}\right) \geqq-4^{N} A_{0} A_{1}
\end{aligned}
$$

by (7). Thus $u^{-}(x)$ is bounded in $\widetilde{B}\left(z_{0}, r_{0}\right)$. Thus $z_{0} \notin Z$, a contradiction based on the assumption that $Z \neq \phi$; thus $Z=\phi$ and Theorem 1 is established.

4. Proof of Theorem 2. The theorem will follow directly from

Step 4. Let $f(y)$ satisfy (3) and set $h(x)=P I(f, x)$. Then $h_{f}(x$, $\rho)$ satisfies (1) and (2).

To see this, set $v(x)=u(x)-h(x)$; then

$$
v(x, \rho)=[u-h](x, \rho) \leqq u_{f}(x, \rho)+h_{f}(x, \rho)
$$

so $v(x, \rho)$ satisfies (1) and (2) since both $u_{f}(x, \rho)$ and $h_{f}(x, \rho)$ do. So by Theorem $1,0 \leqq v(x)$ and thus

$$
v(x)=\int_{\Omega} G\left(x, x^{\prime}\right) d \nu\left(x^{\prime}\right)+g(x)
$$


with all the terms nonnegative. So $g(x, \rho)$ satisfies (1) and (2) and thus $0 \leqq g(x)$; clearly then $0 \leqq-g(x)$ and $g(x) \equiv 0$, whereby (4) follows.

Proof of Step 4. For $y_{0} \in \partial \Omega$, there is a $\gamma$ and a $0<\rho_{0}$ such that

$$
\rho^{1-N} \int_{\overline{\tilde{\partial}}_{B\left(y_{0}, \rho\right)}}\left|f(y)-f\left(y_{0}\right)\right| d y<\gamma \text { for } \rho<\rho_{0} .
$$

Clearly we can assume that $f\left(y_{0}\right)=0$. Consider

$$
\begin{aligned}
&\left|\widetilde{B}\left(y_{0}, \rho\right)\right|^{-1} \int_{\widetilde{B}\left(y_{0}, \rho\right)} \int_{\partial .2}\left\{\left(1-|x|^{2}\right) / \omega_{N}|x-y|^{N}\right\}|f(y)| d \omega_{N}(y) d x \\
&=\int_{\bar{\partial} B\left(y_{0}, 2 \rho\right)}+\int_{\partial \cdot 2-\bar{\partial} B\left(y_{0}, 2, \rho\right)}\left|\widetilde{B}\left(y_{0}, \rho\right)\right|^{-1} \int_{\widetilde{B}\left(y_{0}, \rho\right)}\left(1-|x|^{2}\right) / \omega_{N} \mid x \\
&-\left.y\right|^{N} d x|f(y)| d \omega_{N}(y) \\
&= I_{1}+I_{2} .
\end{aligned}
$$

In the second integral we have $1 / 2\left|y_{0}-y\right| \leqq|x-y| \leqq 2\left|y_{0}-y\right|$, which gives

$$
\begin{aligned}
I_{2} & \leqq A_{1} \rho \int_{\partial \Omega-\bar{\rho} B\left(y_{0}, 2 \rho\right)}|f(y)|\left|y-y_{0}\right|^{-N} d \omega_{v v}(y) \\
& \leqq A_{2 i} O \int_{2 \rho}^{1} r^{-N} \int_{s\left(y_{0}, r\right)}|f(y)| d s_{r}(y) d r
\end{aligned}
$$

where $s\left(y_{0}, r\right)=\partial B\left(y_{0}, r\right) \cap \partial \Omega$

$$
\begin{aligned}
= & \left.A_{2} \rho r^{-v} \int_{0}^{r} \int_{s\left(y_{0}, r^{\prime}\right)}|f(y)| d s_{r^{\prime}}(y) d r^{\prime}\right|_{2 \rho} ^{1} \\
& +A_{2} N \rho \int_{2 \rho}^{\rho_{0}}+\int_{\rho_{0}}^{1}\left\{r^{-N-1} \int_{0}^{r} \int_{s\left(y_{0}, r^{\prime}\right)}|f(y)| d s_{r^{\prime}}(y)\right\} d r^{\prime} \\
\leqq & A_{3} \gamma+o(\rho) \quad \text { as } \rho \longrightarrow 0 .
\end{aligned}
$$

For $I_{1}$ we use the inequality

$$
\int_{\widetilde{B}\left(y_{0}, \rho\right)}\left(1-|x|^{2}\right) / \omega_{N}|x-y|^{N} d x \leqq \int_{\tilde{B}\left(y_{0}, 2 \rho\right)}\left(1-|x|^{2}\right) / \omega_{N}\left|x-y_{0}\right|^{N} d x
$$

to obtain

$$
\begin{aligned}
I_{1} & \leqq A_{1}\left|\widetilde{B}\left(y_{0}, \rho\right)\right|^{-1} \int_{\tilde{B}\left(y_{0}, 2 \rho\right)}\left(1-|x|^{2}\right) /\left|x-y_{0}\right|^{v} d x \cdot \int_{\bar{\partial} B\left(y_{0}, 2 \rho\right)}|f(y)| d y \\
& \leqq A_{2} \rho^{1-x} \int_{\bar{\partial} B\left(y_{0}, 2 \rho\right)}|f(y)| d y \\
& \leqq A_{3} \gamma,
\end{aligned}
$$

which shows that $h_{f}(x, \rho)$ satisfies $(2)$. Since $\gamma$ can be taken arbitrarily small for almost every $y_{0} \in \partial \Omega, h_{f}(x, \rho)$ also satisfies (1). 
5. Converse to Theorem 1. Let $u(x)=\int_{\Omega} G\left(x, x^{\prime}\right) d \eta\left(x^{\prime}\right)$, with $u(x)$ in $L_{1}$ on $\Omega$. Zygmund constructed, see [5, p. 644], such a $u(x)$ which fails to have a finite nontangential limit at every point of the boundary of unit disc. Even so, Tolsted and Solomentseff have established in $R^{2}$ and $R^{N}$ respectively that $u$ must have radial limit zero a.e. along any nontangential ray. However, Zygmund's example as well as the other examples in [5], have a zero mean continuous boundary limit a.e., i.e., they satisfy (2).

Open Question: Is there an $L_{1}$, Green potential which does not satisfy (2) ${ }^{1}$

It is interesting to note that continuity at a boundary point $y_{0}$ implies mean continuity at $y_{0}$ which implies nontangential limit at $y_{0}$ for harmonic functions. From the above examples, we see that this hierarchy fails for superharmonic functions. Furthermore it is not clear that mean continuity at $y_{0}$ implies a radial limit at $y_{0}$ for superharmonic functions.

\section{REFERENCES}

1. V. L. Shapiro, The uniqueness of functions harmonic in the interior of the unit disk, Proc. London. Math. Soc., 13 (1963), 639-52.

2. - Fourier series in several variables, Bull. Amer. Math. Soc., 70 (1964), 48-93.

3. One-sided conditions for functions harmonic in the unit disc, Amer. Math. Soc., Summer Institute on P.D. E. (1971), University of Calif., Berkeley.

4. E. D. Solomentseff, Sur les valeurs limites des functions sousharmoniques, Czech. Math. J., 8 (1958), 535-536 (Resume).

5. E. B. Tolsted, Limiting values of subharmonic functions, Proc. Amer. Math. Soc., 1 (1950), 636-647.

6. - Nontangential limits of subharmonic functions, Proc. London Math. Soc., 7 (1957), 321-333.

University of CALifornia, Davis

1 The answer is negative, i.e., every $L_{1}$ Green potential satisfies (2). See the Notices, Jaw. 1975. 


\section{PACIFIC JOURNAL OF MATHEMATICS}

\section{EDITORS}

RICHARD ARENS (Managing Editor)

University of California

Los Angeles, California 90024

R. A. Beaumont

University of Washington

Seattle, Washington 98105
J. DugundJI

Department of Mathematics University of Southern California Los Angeles, California 90007

D. Gilbarg AND J. Milgram Stanford University

Stanford, California 94305

\section{ASSOCIATE EDITORS}
E. F. BECKENBACH
B. H. NeUmanN
F. WOLF
K. YOSHIDA

\section{SUPPORTING INSTITUTIONS}

UNIVERSITY OF BRITISH COLUMBIA CALIFORNIA INSTITUTE OF TECHNOLOGY

UNIVERSITY OF CALIFORNIA

MONTANA STATE UNIVERSITY

UNIVERSITY OF NEVADA

NEW MEXICO STATE UNIVERSITY

OREGON STATE UNIVERSITY

UNIVERSITY OF OREGON

OSAKA UNIVERSITY
UNIVERSITY OF SOUTHERN CALIFOF

STANFORD UNIVERSITY

UNIVERSITY OF TOKYO

UNIVERSITY OF UTAH

WASHINGTON STATE UNIVERSITY UNIVERSITY OF WASHINGTON

AMERICAN MATHEMATICAL SOCIET NAVAL WEAPONS CENTER 


\section{Pacific Journal of Mathematics}

\section{Vol. 54, No. 1 \\ May, 1974}

Ralph K Amayo, Engel Lie rings with chain conditions ..................

Bernd Anger and Jörn Lembcke, Hahn-Banach type theorems for hypolinear

functionals on preordered topological vector spaces ..................

Gregory Frank Bachelis and Samuel Ebenstein, On $\Lambda(p)$ sets ................

Harvey Isaac Blau, Indecomposable modules for direct products of finite

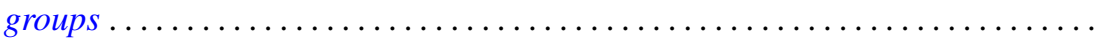

Larry Eugene Bobisud and James Calvert, Singular perturbation of a

time-dependent Cauchy problem in a Hilbert space ................

Walter D. Burgess and Robert Raphael, Abian's order relation and orthogonal

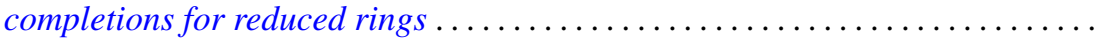

James Diederich, Representation of superharmonic functions mean continuous at

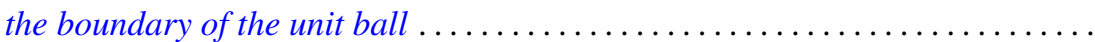

Aad Dijksma and Hendrik S. V. de Snoo, Self-adjoint extensions of symmetric

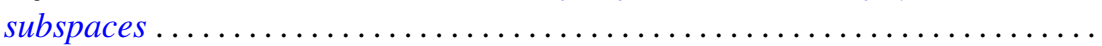

Gustave Adam Efroymson, A Nullstellensatz for Nash rings . . . . . . . . . . . . .

John D. Elwin and Donald R. Short, Branched immersions onto compact orientable surfaces . . . . . . . . . . . . . . . . . . . . . . . . .

John Douglas Faires, Comparison of the states of closed linear

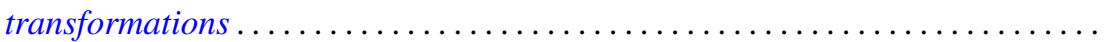

Joe Wayne Fisher and Robert L. Snider, On the von Neumann regularity of rings with regular prime factor rings .

Franklin Takashi Iha, A unified approach to boundary value problems on compact intervals

Palaniappan L. Kannappan and Che Tat $\mathrm{Ng}$, On functional equations connected with directed divergence, inaccuracy and generalized directed divergence

Samir A. Khabbaz and Elias Hanna Toubassi, The module structure of Ext $(F, T)$ over the endomorphism ring of $T$...

Garo K. Kiremidjian, On deformations of complex compact manifolds with boundary.

Dimitri Koutroufiotis, Mappings by parallel normals preserving principal

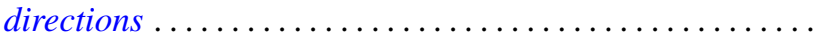

W. K. Nicholson, Semiperfect rings with abelian adjoint group

Norman R. Reilly, Extension of congruences and homomorphisms to translational hulls

Sadahiro Saeki, Symmetric maximal ideals in $M(G)$

Brian Kirkwood Schmidt, On the homotopy invariance of certain functors ...

H. J. Shyr and T. M. Viswanathan, On the radicals of lattice-ordered rings ...

Indranand Sinha, Certain representations of infinite group algebras ...

David Smallen, The group of self-equivalences of certain complexes ...

Kalathoor Varadarajan, On a certain problem of realization in homotopy

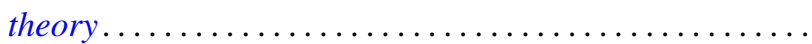

